\title{
ZFAS1 knockdown inhibits fibroblast-like synoviocyte proliferation, migration, invasion and inflammation, and promotes apoptosis via miR-3926/FSTL1 in rheumatoid arthritis
}

\author{
QIANG WANG ${ }^{1,2}$, PEIGANG CHU ${ }^{2}, \mathrm{XIA} \mathrm{YU}^{3}, \mathrm{JUN} \mathrm{LI}^{2}$, WENZHENG ZHANG $^{2}$ and MINGZHI GONG ${ }^{1}$ \\ ${ }^{1}$ Department of Traumatic Orthopaedics, The Second Hospital of Shandong University, \\ Jinan, Shandong 250033; Departments of ${ }^{2}$ Joint Sports Medicine and ${ }^{3}$ Nuclear Medicine, \\ Taian City Central Hospital, Taian, Shandong 271000, P.R. China
}

Received September 25, 2019; Accepted March 31, 2021

DOI: $10.3892 / \mathrm{etm} .2021 .10346$

\begin{abstract}
Rheumatoid arthritis (RA) is a chronic systemic autoimmune disease characterized by joint disorders. Long non-coding RNA zinc finger antisense 1 (ZFAS1) is aberrantly expressed in numerous human diseases, including RA. The present study aimed to investigate the functions and underlying mechanisms of ZFAS1 in RA. Reverse transcription-quantitative PCR was performed to determine the expression levels of ZFAS1, microRNA (miR)-3926 and follistatin-like protein 1 (FSTL1). MTT assay, flow cytometric analysis and Transwell assay were performed to examine the proliferation, apoptosis, migration and invasion of fibroblast-like synoviocytes (FLSs), respectively. Western blotting was employed to measure the protein expression levels of cleaved caspase-3, interleukin (IL)- 6 , IL-1 $\beta$, tumor necrosis factor- $\alpha$ and FSTL1. Dual-luciferase reporter assay was performed to verify the interaction between miR-3926 and ZFAS1 or FSTL1. The results demonstrated that ZFAS1 and FSTL1 were upregulated, and miR-3926 was downregulated in RA synovial tissues and RA-FLSs. ZFAS1 knockdown suppressed cell proliferation, migration, invasion and inflammatory cytokine production, and induced apoptosis in RA-FLSs. ZFAS1 acted as a sponge for miR-3926, and ZFAS1 overexpression abolished the impact of miR-3926 on the development of RA-FLSs. FSTL1 was a direct target of miR-3926, and the effect of FSTL1 knockdown on the progression of RA-FLSs was rescued by miR-3926 inhibition. Furthermore, ZFAS1 regulated FSTL1 expression levels via sponging miR-3926 in RA-FLSs. In conclusion, ZFAS1 knockdown inhibited RA-FLS proliferation, migration,
\end{abstract}

Correspondence to: Dr Mingzhi Gong, Department of Traumatic Orthopaedics, The Second Hospital of Shandong University, 247 Beiyuan Street, Jinan, Shandong 250033, P.R. China

E-mail:vugmdu@163.com

Key words: rheumatoid arthritis, zinc finger antisense 1, microRNA-3926, follistatin-like protein 1, fibroblast-like synoviocytes invasion and inflammatory cytokine production, and induced apoptosis in RA via the miR-3926/FSTL1 axis.

\section{Introduction}

Rheumatoid arthritis (RA) is an autoimmune disease characterized by chronic and progressive joint inflammation, which can lead to irreversible destruction of articular cartilage, eventually leading to joint deformity and physical disability $(1,2)$. It has been revealed that the pathophysiological behaviors of fibroblast-like synoviocytes (FLSs) serve crucial roles in the pathogenesis of RA (3). Abnormal secretion of inflammatory cytokines is also an important cause of RA $(4,5)$. However, the pathogenesis of RA remains ill-defined.

Long non-coding RNAs (lncRNAs) are a class of ncRNA $>200$ nucleotides (nts) long without protein-coding ability, which have key roles in numerous biological processes, such as cell differentiation, proliferation, migration and apoptosis $(6,7)$. Evidence has revealed that IncRNAs are associated with the development of RA; for instance, Zou et al (8) demonstrated that one lncRNA, LERFS, was decreased in RA, and that its elevation notably hindered RA progression via inhibiting the proliferation, migration and invasion of FLSs. Yue et al (9) reported that ITSN1-2 was highly expressed in RA-FLSs, and that its depletion hampered RA-FLS growth and inflammation, as well as inducing apoptosis in RA. These data indicated that lncRNAs may serve dual roles in RA progression. A previous report confirmed that zinc finger antisense 1 (ZFAS1) contributed to the migration and invasion of RA-FLSs in RA (10). However, the functions and mechanisms of ZFAS1 have not been fully elucidated.

MicroRNAs (miRNAs/miRs) are a set of endogenous ncRNAs 22 nts in length, which modulate gene expression at the post-transcriptional level (11). miRNAs are also involved in the regulation of numerous biological processes, and multiple studies have focused on the roles of miRNAs in human diseases, including RA (12). For example, Philippe et al (13) demonstrated that miR-20a was dysregulated and affected the release of pro-inflammatory cytokines in RA. Wang et al (14) revealed that miR-573 decelerated the progression of RA and may be a target for RA treatment. miR-410-3p has also been 
shown to suppress cell viability and facilitate apoptosis in RA-FLSs (15). A previous report revealed that miR-3926 was decreased in the plasma of patients with RA (16). Nevertheless, knowledge about the underlying mechanisms of miR-3926 in RA is limited.

Follistatin-like protein 1 (FSTL1), also referred to as follistatin-related protein gene, is a transforming growth factor $\beta 1$-inducible protein, containing follistatin-like and extracellular calcium-binding domains, which is associated with RA development (17). FSTL1 has been reported to modulate inflammatory cytokine secretion and FLS migration and invasion in RA $(18,19)$. However, to the best of our knowledge, there are no reports about whether miR-3926 interacts with FSTL1 in RA.

The present study investigated the expression level patterns of ZFAS1, miR-3926 and FSTL1 in RA. Furthermore, the roles and mechanisms of ZFAS1, miR-3926 and FSTL1 in RA were further investigated by gain- and loss-of-function experiments.

\section{Materials and methods}

Tissue collection. A total of 20 RA synovial tissues were harvested from patients with RA (13 women and 7 men; age range, 41-66 years) who underwent knee arthroplasty, and 20 non-arthritic control tissues were collected from the knee joints of traumatic amputees (11 women and 9 men; age range, 38-62 years) at the Second Hospital of Shandong University (Jinan, China) between May 2013 and July 2018. RA was defined and classified according to the 2010 American College of Rheumatology/European League against Rheumatism classification criteria for RA (20). The experiments were approved by the Ethics Committee of the Second Hospital of Shandong University and written informed consent was provided by all participants. The specimens were preserved at $-80^{\circ} \mathrm{C}$ for the isolation of total RNA or protein.

Cell culture. FLSs were isolated from synovial tissues. After being washed with PBS (Beijing Solarbio Science \& Technology, Co., Ltd.) and serum-free DMEM (HyClone; Cytiva), synovial tissues were cut into small sections $\left(1 \mathrm{~mm}^{3}\right)$ and digested with $2 \mathrm{mg} / \mathrm{ml}$ collagenase I (Beijing Solarbio Science \& Technology, Co., Ltd.) for $2 \mathrm{~h}$ at $37^{\circ} \mathrm{C}$. The precipitate was resuspended in DMEM containing 10\% FBS (both HyClone; Cytiva) following centrifugation at $200 \mathrm{x}$ g for $10 \mathrm{~min}$ at $37^{\circ} \mathrm{C}$. FLSs were harvested and grown in DMEM containing $10 \% \mathrm{FBS}$ and $1 \%$ penicillin-streptomycin (all HyClone; Cytiva) at $37^{\circ} \mathrm{C}$ in a humidified atmosphere with $5 \% \mathrm{CO}_{2}$. FLSs at passages 3-6 were used for further experiments.

Cell transfection. Small interfering RNA (siRNA) against ZFAS1 (si-ZFAS1; 5'-ACA AAACGGGAACTTACG GGC-3'), siRNA against FSTL1 (si-FSTL1; 5'-AGTAAA TATCCTAGTTTTCTC-3') and scrambled siRNA negative control (si-NC; 5'-TTCTCCGAACGTGTCACGTTT-3'); the overexpression vector of ZFAS1 (pcDNA-ZFAS1) and empty pcDNA (pcDNA-NC); miR-3926 mimics (miR-3926; 5'-UGGCCAAAAAGCAGGCAGAGA-3') and scrambled miRNA control (miR-NC; 5'-UUCUCCGAACGUGUC ACGUUU-3'); miR-3926 inhibitors (anti-miR-3926; 5'-UCU CUGCCUGCUUUUUGGCCA-3') and inhibitor control
(anti-miR-NC; 5'-CAGUACUUUUGUGUAGUACAA-3') were all obtained from Guangzhou RiboBio Co., Ltd. RA-FLSs $\left(1.0 \times 10^{4}\right.$ cells/well) were seeded into 24-well plates and transfected with the oligonucleotides $(50 \mathrm{nM})$ or vectors $(2 \mu \mathrm{g})$ using Lipofectamine ${ }^{\circledR} 2000$ (Invitrogen; Thermo Fisher Scientific, Inc.). For miR-3926 + pcDNA-NC and miR-3926 + pcDNA-ZFAS1 groups, cells were co-transfected with miR-3926 and pcDNA-NC/pcDNA-ZFAS1. For si-FSTL1 + anti-miR-NC or si-FSTL1 + anti-miR-3926 groups, cells were co-transfected with si-FSTL1 and anti-miR-NC/anti-miR-3926. After transfection for $48 \mathrm{~h}$, the cells were collected for subsequent experiments.

Reverse transcription quantitative $(R T-q) P C R$. Total RNA in synovial tissues and FLSs was extracted using RNAiso Plus (Takara Biotechnology Co., Ltd.). Subsequently, RNA was quantified using a NanoDrop 2000c spectrophotometer (Thermo Fisher Scientific, Inc.). RT was conducted using M-MLV First Strand (Takara Biotechnology Co., Ltd.) and miRNA 1st Strand cDNA Synthesis kits (Vazyme Biotech Co., Ltd.) according to the manufacturers' instructions. RT-qPCR was then performed using BeyoFast ${ }^{\mathrm{TM}}$ SYBR Green qPCR Mix (Beyotime Institute of Biotechnology) on an ABI 7900 Real-Time PCR system (Applied Biosystems; Thermo Fisher Scientific, Inc.) under the following conditions: Initial denaturation at $95^{\circ} \mathrm{C}$ for $5 \mathrm{~min} ; 40$ cycles at $95^{\circ} \mathrm{C}$ for $10 \mathrm{sec}$ and $60^{\circ} \mathrm{C}$ for $30 \mathrm{sec} ; 95^{\circ} \mathrm{C}$ for $15 \mathrm{sec}, 60^{\circ} \mathrm{C}$ for $60 \mathrm{sec}$ and $95^{\circ} \mathrm{C}$ for $15 \mathrm{sec}$. The expression levels of ZFAS1, FSTL1 and miR-3926 were analyzed via the $2^{-\Delta \Delta \mathrm{Cq}}$ method (21). GAPDH was used as the internal reference for ZFAS1 and FSTL1, while small nuclear RNA U6 was utilized as the internal reference for miR-3926. The primer sequences were as follows: ZFAS1, forward 5'-CTA TTGTCCTGCCCGTTAGAG-3' and reverse 5'-GTCAGG AGATCGAAGGTTGTAG-3'; miR-3926, forward 5'-GCC GAGTGGCCAAAAAGCA-3' and reverse 5'-CTCAACTGG TGTCGTGGA-3'; FSTL1, forward 5'-GAGCAATGCAAA CCTCACAAG-3' and reverse 5'-CAGTGTCCATCGTAA TCAACCTG-3'; GAPDH, forward 5'-GATTCCACCCAT GGCAAATTC-3' and reverse 5'-CTGGAAGATGGTGAT GGGATT-3'; and U6, forward 5'-CTTCGGCAGCACATA TACT-3' and reverse 5'-AAAATATGGAACGCTTCACG-3'.

MTT assay. After transfection for $48 \mathrm{~h}, \mathrm{RA}-\mathrm{FLSs}$ were collected and seeded into 96 -well plates $\left(5.0 \times 10^{3}\right.$ cells/well). Subsequently, $20 \mu \mathrm{l}$ MTT (Beijing Solarbio Science \& Technology, Co., Ltd.) was added to each well at the indicated time points $(0,24,48$ or $72 \mathrm{~h})$ and incubated for an additional $4 \mathrm{~h}$ at $37^{\circ} \mathrm{C}$. DMSO (150 $\mu \mathrm{l}$; Beijing Solarbio Science $\&$ Technology, Co., Ltd.) was then added to dissolve the formazan crystals. Finally, the absorbance at $490 \mathrm{~nm}$ was read via a microplate reader (Molecular Devices, LLC).

Flow cytometric analysis. After transfection, Annexin V-FITC/propidium iodide (PI) Apoptosis Detection kit (Beyotime Institute of Biotechnology) was utilized to evaluate apoptosis of RA-FLSs. Briefly, transfected RA-FLSs were harvested and resuspended in binding buffer at a density of $1.0 \times 10^{6}$ cells $/ \mathrm{ml}$. Subsequently, RA-FLSs were maintained in $5 \mu \mathrm{l}$ Annexin V-FITC and $10 \mu \mathrm{l}$ PI for $15 \mathrm{~min}$ at room temperature in the dark. Finally, a FACScan ${ }^{\circledR}$ flow cytometer 
(BD Biosciences) was used to assess cell apoptosis. The results were analyzed by FlowJo 7.6.1. (FlowJo LLC).

Western blotting. Total protein was isolated by lysing synovial tissues and FLSs in RIPA buffer (Beyotime Institute of Biotechnology). The protein samples were quantified using a NanoDrop 2000 spectrophotometer (Thermo Fisher Scientific, Inc.) by examining the OD value at $280 \mathrm{~nm}$. Equal amounts of protein $(25 \mu \mathrm{g} /$ lane $)$ were separated by 10\% SDS-PAGE (Beijing Solarbio Science \& Technology, Co., Ltd.) and transferred onto PVDF membranes (Pall Corporation). Subsequently, the membranes were blocked in 5\% skimmed milk for $2 \mathrm{~h}$ at room temperature and incubated overnight at $4{ }^{\circ} \mathrm{C}$ with primary antibodies (all BIOSS) against cleaved caspase-3 (C-caspase-3; cat. no. bs-0081R; 1:2,000), interleukin (IL)-6 (cat. no. bs-4539R; 1:2,000), IL-1 $\beta$ (cat. no. bs-20448R; 1:2,000), tumor necrosis factor (TNF)- $\alpha$ (cat. no. bs-10802R; 1:2,000), FSTL1 (cat. no. bs-6050R; 1:2,000) and GAPDH (cat. no. bs-0755R; 1:5,000), followed by incubation with a horseradish peroxidase-conjugated secondary antibody (cat. no. bs-40296G-HRP; 1:10,000; BIOSS) for $1 \mathrm{~h}$ at room temperature. The bands were visualized via enhanced chemiluminescence reagent (Vazyme Biotech Co., Ltd.) and analyzed using ImageJ v1.8.0 (National Institutes of Health).

Transwell assay. For the detection of cell migration, Transwell insert chambers (Corning Inc.) were used. Briefly, transfected RA-FLSs $\left(5.0 \times 10^{5}\right.$ cells $\left./ \mathrm{ml}\right)$ were suspended in serum-free DMEM in the upper chamber, and DMEM (600 $\mu \mathrm{l})$ containing $10 \%$ FBS (HyClone; Cytiva) was added into the bottom chamber. After $24 \mathrm{~h}$, migrated cells were fixed in $75 \%$ methanol for $1 \mathrm{~h}$ at $37^{\circ} \mathrm{C}$ and stained with $0.1 \%$ crystal violet (Beijing Solarbio Science \& Technology, Co., Ltd.) for $15 \mathrm{~min}$ at $37^{\circ} \mathrm{C}$. The stained cells were analyzed under a light microscope (Olympus Corporation). The same method was used to detect cell invasion, with the exception that the upper chamber was pre-coated with Matrigel (Beijing Solarbio Science \& Technology, Co., Ltd.).

Dual-luciferase reporter assay. The binding sites between ZFAS1 and miR-3926 were predicted using the online software LncBase Predicted V.2 (http://carolina.imis.athena-innovation.gr/diana tools/web/index.php?r=lncbasev2/index-predicted), and the binding sites between miR-3926 and FSTL1 were predicted using TargetScan (http://www.targetscan.org/vert_71/). Subsequently, dual-luciferase reporter assays were used to verify these predictions. The sequences of ZFAS1 or FSTL1 3'untranslated region (UTR) including wild-type (WT) or mutant (MUT) binding sequences of miR-3926 were cloned and inserted into pmirGLO vectors (Promega Corporation), in order to generate ZFAS1 WT, ZFAS1 MUT, FSTL1 3'UTR WT and FSTL1 3'UTR MUT luciferase reporter vectors, respectively. Subsequently, RA-FLSs ( $2.0 \times 10^{4}$ cells/well) were seeded into 24 -well plates and co-transfected with miR-3926 or miR-NC and corresponding luciferase reporter vectors using Lipofectamine ${ }^{\circledR} 2000$ (Invitrogen; Thermo Fisher Scientific, Inc.) according to the manufacturers' instructions. After co-transfection for $48 \mathrm{~h}$, luciferase activity was evaluated using a dual-luciferase reporter assay kit (Promega Corporation) according to the manufacturers' instructions. Renilla luciferase activity was used to normalize firefly luciferase activity.
Statistical analysis. The data were analyzed using GraphPad Prism 7 software (GraphPad, Inc.) and are presented as the mean \pm standard deviation of three independent experiments. Significant differences between two groups were analyzed by unpaired Student's t-test, whereas those between three groups were analyzed by one-way ANOVA followed by Tukey's post hoc test. The linear associations between ZFAS1, miR-3926 and FSTL1 in RA tissues were analyzed by Pearson's correlation analysis. $\mathrm{P}<0.05$ was considered to indicate a statistically significant difference.

\section{Results}

ZFAS1 is highly expressed in RA synovial tissue and RA-FLSs. Firstly, the expression levels of ZFAS1 in RA and healthy synovial tissues were evaluated via RT-qPCR. ZFAS1 expression levels were markedly elevated in RA synovial tissue compared with those in non-arthritic control tissue (Fig. 1A). Additionally, the expression levels of ZFAS1 in RA-FLSs and non-arthritic control tissue-extracted FLSs (N-FLSs) were measured. The data demonstrated that ZFAS1 was more highly expressed in RA-FLSs than in N-FLSs (Fig. 1B). These findings indicated that ZFAS1 was abnormally expressed in RA.

Silencing of ZFAS1 inhibits cell proliferation, migration, invasion and inflammatory cytokine expression, and promotes cell apoptosis in RA-FLSS. In order to identify the potential roles of ZFAS1 in RA, loss-of-function experiments were performed via transfecting si-ZFAS1 into RA-FLSs. The knockdown efficiency was determined via RT-qPCR, which demonstrated that ZFAS1 expression levels were significantly decreased in RA-FLSs following si-ZFAS1 transfection (Fig. 2A). The MTT assay indicated that si-ZFAS1 transfection significantly suppressed cell proliferation in RA-FLSs compared with that in cells transfected with si-NC (Fig. 2B). Flow cytometric analysis demonstrated that compared with in the si-NC group, cell apoptosis was significantly induced in RA-FLSs transfected with si-ZFAS1 (Fig. 2C). Furthermore, the expression levels of the apoptosis-associated protein C-caspase-3 were increased in RA-FLSs transfected with si-ZFAS1, as indicated by western blotting (Fig. 2D). Transwell assay demonstrated that ZFAS1 knockdown significantly hampered cell migration and invasion in RA-FLSs (Fig. 2E and F). In addition, the expression levels of inflammatory cytokines, IL- 6 , IL- $1 \beta$ and TNF- $\alpha$, were examined by western blotting. The data showed that the expression levels of IL-6, IL-1 $\beta$ and TNF- $\alpha$ were decreased in RA-FLSs post-transfection with si-ZFAS1 compared with in the si-NC group (Fig. 2G). Taken together, these data indicated that ZFAS1 knockdown suppressed the progression of RA.

ZFAS1 negatively modulates miR-3926 expression levels by directly targeting miR-3926 in RA-FLSs. In order to investigate the mechanism underlying the effects of ZFAS1 on RA, online software LncBase Predicted V.2 was utilized to predict the potential targets of ZFAS1. A total of four miRNAs (miR-548a-3p, miR-3926, miR-27a-3p and miR-4701-5p) were selected for their low expression in RA. Subsequently, RT-qPCR was used to determine the expression levels of these miRNAs in RA-FLSs following ZFAS1 knockdown or 
A

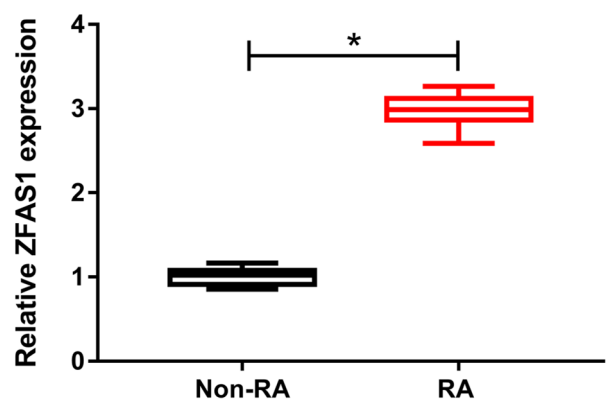

B

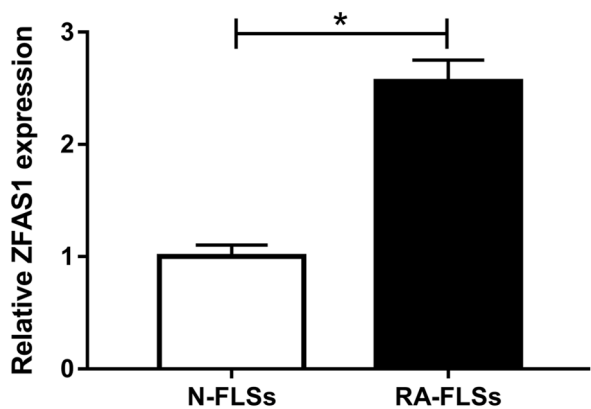

Figure 1. ZFAS1 expression levels are significantly elevated in RA synovial tissues and RA-FLSs. Expression levels of ZFAS1 in (A) RA synovial tissue and non-arthritic control tissue, and (B) RA-FLSs and N-FLSs were analyzed via reverse transcription-quantitative PCR. "P<0.05. ZFAS1, zinc finger antisense 1; RA, rheumatoid arthritis; FLSs, fibroblast-like synoviocytes; N, normal.
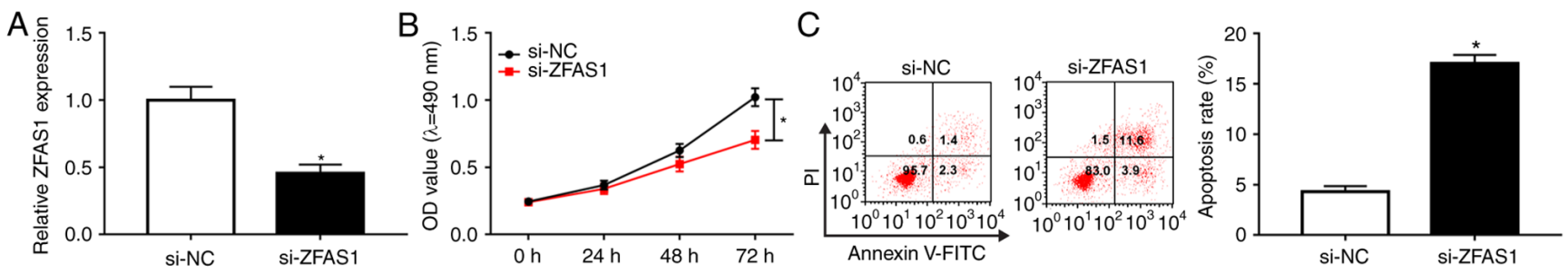

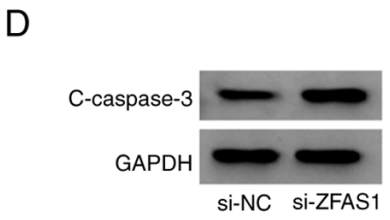

$\mathrm{F}$

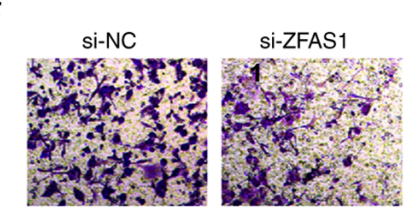

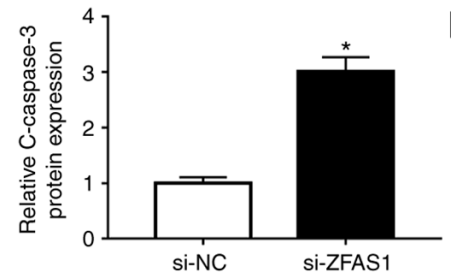

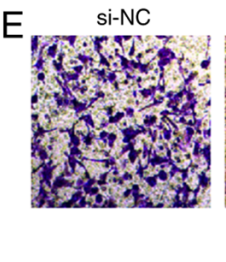

si-ZFAS1

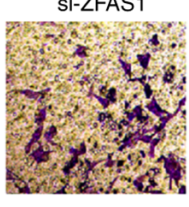

G
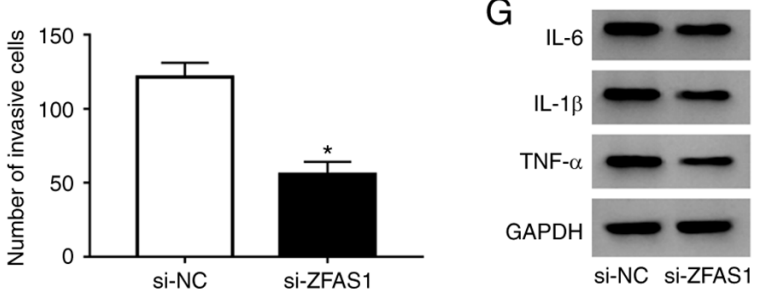
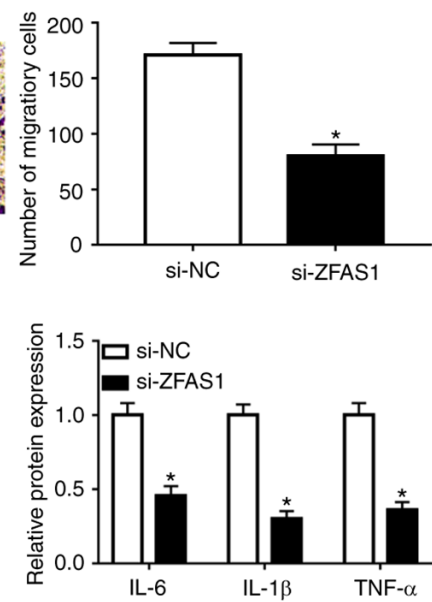

Figure 2. ZFAS1 downregulation suppresses cell proliferation, migration, invasion and inflammatory cytokine expression, and facilitates cell apoptosis in RA-FLSs. RA-FLSs were transfected with si-NC or si-ZFAS1. (A) Expression levels of ZFAS1 were detected via reverse transcription-quantitative PCR. (B) Proliferation of RA-FLSs was evaluated via MTT assay. (C) Apoptosis of RA-FLSs was assessed via flow cytometric analysis. (D) Protein expression levels of C-caspase-3 were measured via western blotting. (E) Migration and (F) invasion of RA-FLSs were examined via Transwell assay (magnification, x100). (G) Expression levels of IL-6, IL-1 $\beta$ and TNF- $\alpha$ were measured via western blotting. ${ }^{*} \mathrm{P}<0.05$ vs. si-NC. ZFAS1, zinc finger antisense 1 ; RA-FLSs, rheumatoid arthritis-fibroblast-like synoviocytes; si, small interfering RNA; NC, negative control; C-caspase-3, cleaved-caspase-3; IL, interleukin; TNF- $\alpha$, tumor necrosis factor- $\alpha$; PI, propidium iodide.

overexpression. miR-3926 was selected as the study object for its marked change in RA-FLSs following ZFAS1 knockdown or ZFAS1 overexpression compared with other miRNAs (Table SI). The complementary sequences between ZFAS1 and miR-3926 are presented in Fig. 3A. To verify these predictions, a dual-luciferase reporter assay was performed. The results demonstrated that the luciferase activity in RA-FLSs co-transfected with ZFAS1 WT and miR-3926 was significantly inhibited compared with that in RA-FLSs co-transfected with ZFAS1 WT and miR-NC, whereas the luciferase activity was not affected in the ZFAS1 MUT group (Fig. 3B). Subsequently, the expression levels of miR-3926 in RA synovial tissue and RA-FLSs were determined via RT-qPCR. The results indicated that miR-3926 expression levels were significantly decreased in RA synovial tissue and RA-FLSs compared with those in non-arthritic control tissue and N-FLSs (Fig. 3C and D). As evidenced by Pearson's correlation analysis, miR-3926 expression levels were inversely correlated with ZFAS1 expression levels in RA synovial tissue (Fig. 3E).

pcDNA-ZFAS1 was successfully transfected into RA-FLSs to elevate the expression levels of ZFAS1 (Fig. 3F). ZFAS1 depletion markedly enhanced the expression levels of miR-3926, whereas ZFAS1 overexpression resulted in decreased expression levels of miR-3926 in RA-FLSs (Fig. 3G). miR-3926 inhibitor transfection also significantly downregulated the expression levels of miR-3926 in RA-FLSs, which indicated that the miR-3926 inhibitor was successfully transfected into RA-FLSs (Fig. 3H). These data indicated 
A

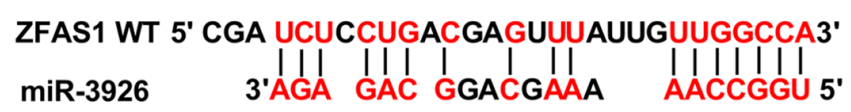

ZFAS1 MUT 5'CGAAGACGACAGGACUAAAUUGAACCGGU 3'
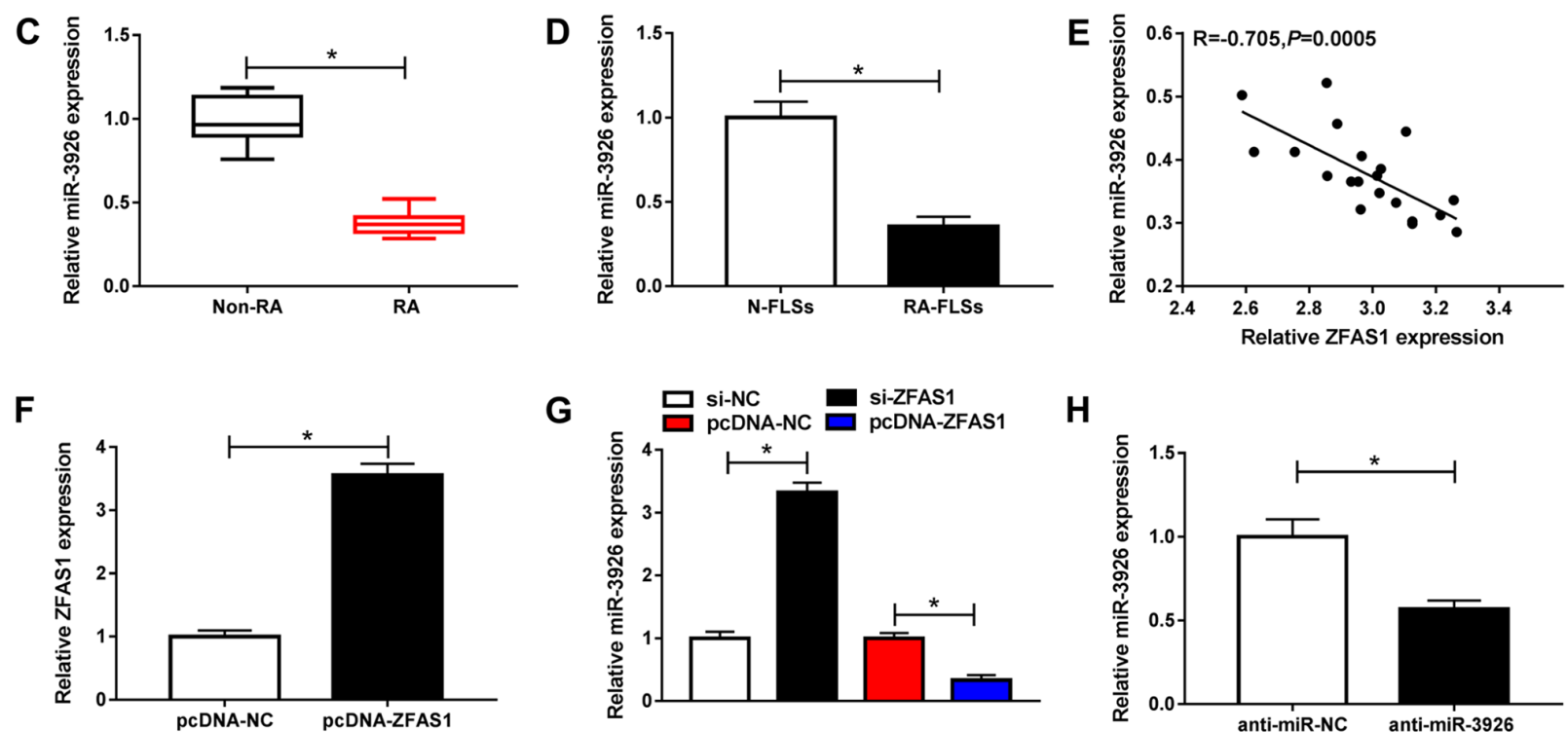

Figure 3. ZFAS1 directly binds miR-3926 and negatively regulates miR-3926 expression levels in RA-FLSs. (A) Predicted binding sites between ZFAS1 and miR-3926. (B) Luciferase activity in RA-FLSs transfected with ZFAS1 WT or ZFAS1 MUT together with miR-3926 or miR-NC was analyzed via dual-luciferase reporter assay. Expression levels of miR-3926 in (C) RA synovial and non-arthritic control tissue, and (D) RA-FLSs and N-FLSs were assessed via RT-qPCR. (E) Correlation between expression levels of ZFAS1 and miR-3926 in RA-FLSs was analyzed via Pearson's correlation analysis. (F) Expression levels of ZFAS1 in RA-FLSs transfected with pcDNA-ZFAS1 or pcDNA-NC was examined via RT-qPCR analysis. (G) RA-FLSs were transfected with si-NC, si-ZFAS1, pcDNA-NC or pcDNA-ZFAS1, then the expression levels of miR-3926 were determined via RT-qPCR. (H) Expression levels of miR-3926 in RA-FLSs transfected with anti-miR-NC or anti-miR-3926 were determined via RT-qPCR. "P<0.05 vs. miR-NC. ZFAS1, zinc finger antisense 1 ; miR, microRNA; RA-FLSs, rheumatoid arthritis-fibroblast-like synoviocytes; WT, wild type; MUT, mutant; NC, negative control; N, normal; RT-qPCR, reverse transcription-quantitative PCR; si, small interfering RNA.

that ZFAS1 may directly interact with and negatively regulate miR-3926 expression in RA-FLSs.

Overexpression of ZFAS1 abolishes effects of miR-3926 overexpression on cell proliferation, apoptosis, migration, invasion and inflammatory cytokine production in RA-FLSs. Given that the present study suggested that ZFAS1 may target miR-3926 to modulate miR-3926 expression in RA-FLSs, the present study investigated whether ZFAS1 could regulate the promotion of RA via targeting miR-3926. RA-FLSs were transfected with miR-NC, miR-3926, miR-3926 + pcDNA-NC or miR-3926 + pcDNA-ZFAS1, and miR-3926 expression levels were measured via RT-qPCR. Overexpression of miR-3926 caused by miR-3926 transfection was partly abolished by pcDNA-ZFAS1 transfection in RA-FLSs (Fig. 4A). miR-3926 overexpression led to a marked inhibition in cell proliferation in RA-FLSs, whereas this inhibition was partially reversed following the overexpression of ZFAS1, as evidenced by the MTT assay (Fig. 4B). Flow cytometric analysis indicated that the apoptotic rate of RA-FLSs was significantly increased by miR-3926, whereas pcDNA-ZFAS1 counteracted this effect (Fig. 4C). Furthermore, the expression levels of the pro-apoptotic protein C-caspase3 were markedly elevated by miR-3926 overexpression; however, ZFAS1 overexpression significantly weakened this elevation in RA-FLSs (Fig. 4D). As demonstrated by the Transwell assay, there was a significant decrease in the number of migratory and invasive cells transfected with miR-3926; this decrease was rescued following transfection with pcDNA-ZFAS1 in RA-FLSs (Fig. 4E and F). The results of western blotting demonstrated that miR-3926 overexpression suppressed the production of IL-6, IL-1 $\beta$ and TNF- $\alpha$ in RA-FLSs, whereas ZFAS1 overexpression partly attenuated the effects in RA-FLSs (Fig. 4G). These data indicated that the inhibitory effect of miR-3926 on RA development was markedly overturned by ZFAS1.

FSTL1 is a direct target of miR-3926 in RA-FLSs. Using TargetScan online software, FSTL1 was identified as a target gene of miR-3926 (Fig. 5A). Dual-luciferase reporter assay revealed a significant suppression in the luciferase activity of RA-FLSs co-transfected with FSTL1 3'UTR WT and miR-3926 compared with the FSTL1 3'UTR WT and miR-NC co-transfected group, whereas the luciferase activity was not affected in the FSTL1 3'UTR MUT group (Fig. 5B). The mRNA and protein expression levels of FSTL1 in RA synovial tissue and healthy control tissue were determined 


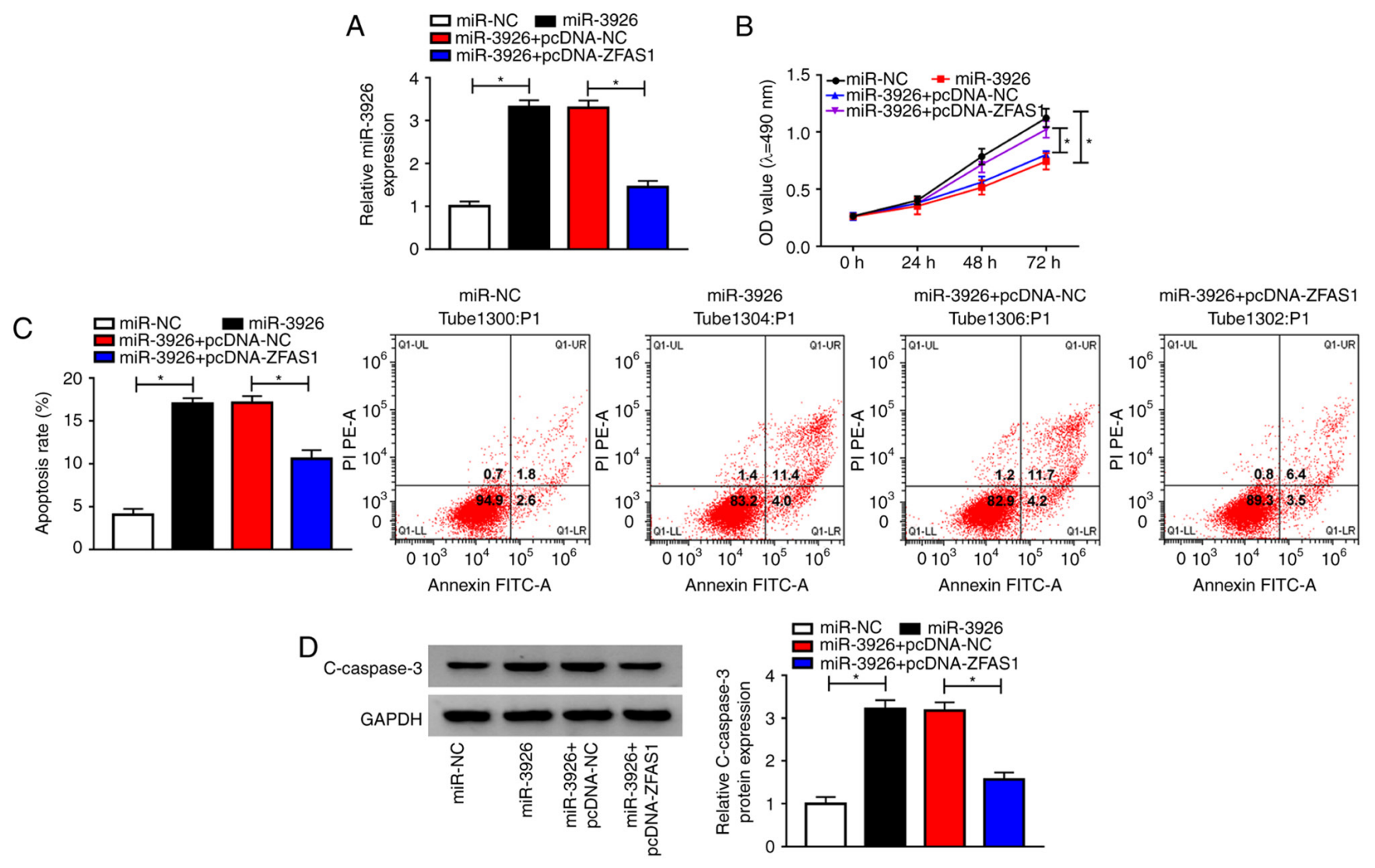

\section{E miR-3926+pcDNA-ZFAS1}

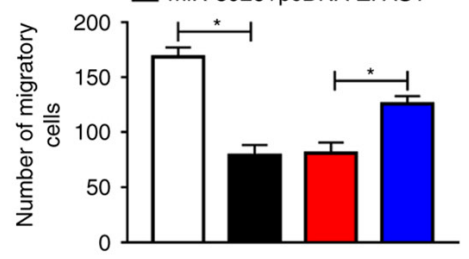

$\mathrm{F}$

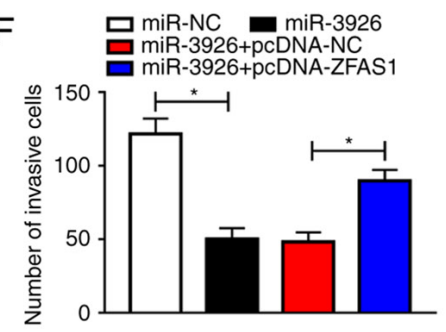

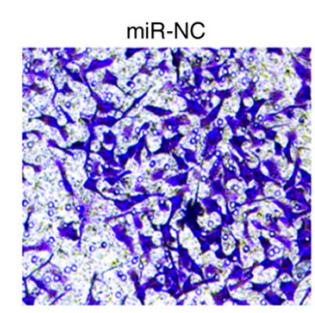
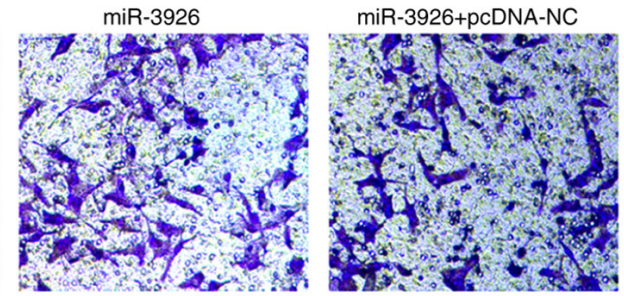

miR-3926+pcDNA-ZFAS1

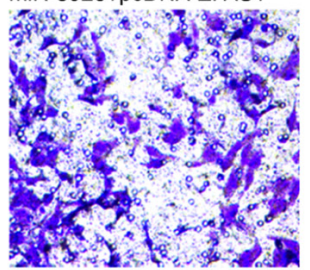

miR-NC

$\operatorname{miR}-3926$
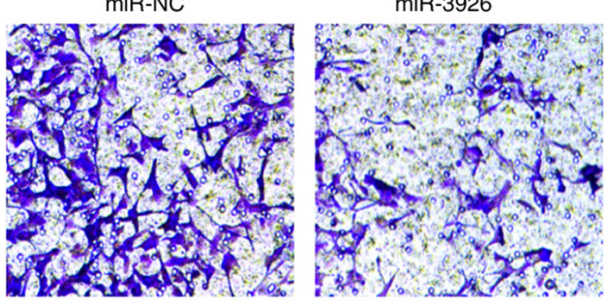

miR-3926+pcDNA-NC

miR-3926+pcDNA-ZFAS1
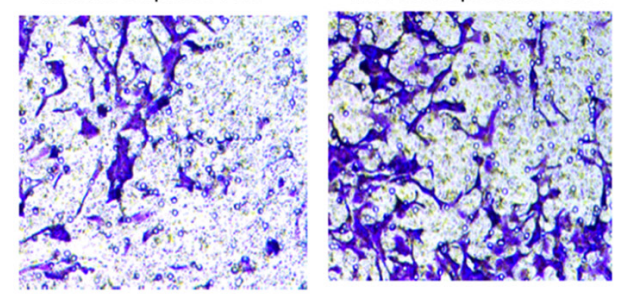

G
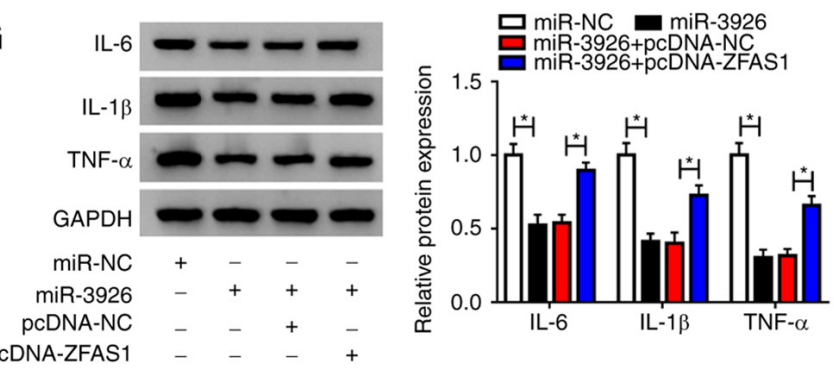

Figure 4.ZFAS1 alters cell proliferation, apoptosis, migration, invasion and inflammatory cytokine expression via binding to miR-3926 in RA-FLSs. RA-FLSs were transfected with miR-NC, miR-3926, miR-3926 + pcDNA-NC or miR-3926 + pcDNA-ZFAS1. (A) Expression levels of miR-3926 in RA-FLSs were measured via reverse transcription-quantitative PCR. (B) RA-FLS proliferation was determined via MTT assay. (C) RA-FLS apoptosis was analyzed via flow cytometric analysis. (D) Expression levels of C-caspase-3 in RA-FLSs were determined via western blotting. (E) Migration and (F) invasion of RA-FLS were assessed via Transwell assay (magnification, x100). (G) Expression levels of IL-6, IL-1 $\beta$ and TNF- $\alpha$ were determined via western blotting assay. ${ }^{*} \mathrm{P}<0.05$. ZFAS1, zinc finger antisense 1; miR, microRNA; RA-FLSs, rheumatoid arthritis-fibroblast-like synoviocytes; NC, negative control; C-caspase-3, cleaved-caspase-3; IL, interleukin; TNF- $\alpha$, tumor necrosis factor- $\alpha$. 
A

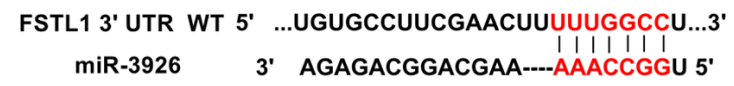

FSTL1 3' UTR MUT 5' ...UGUGCCUUCGAACUUAAACCGGU...3'
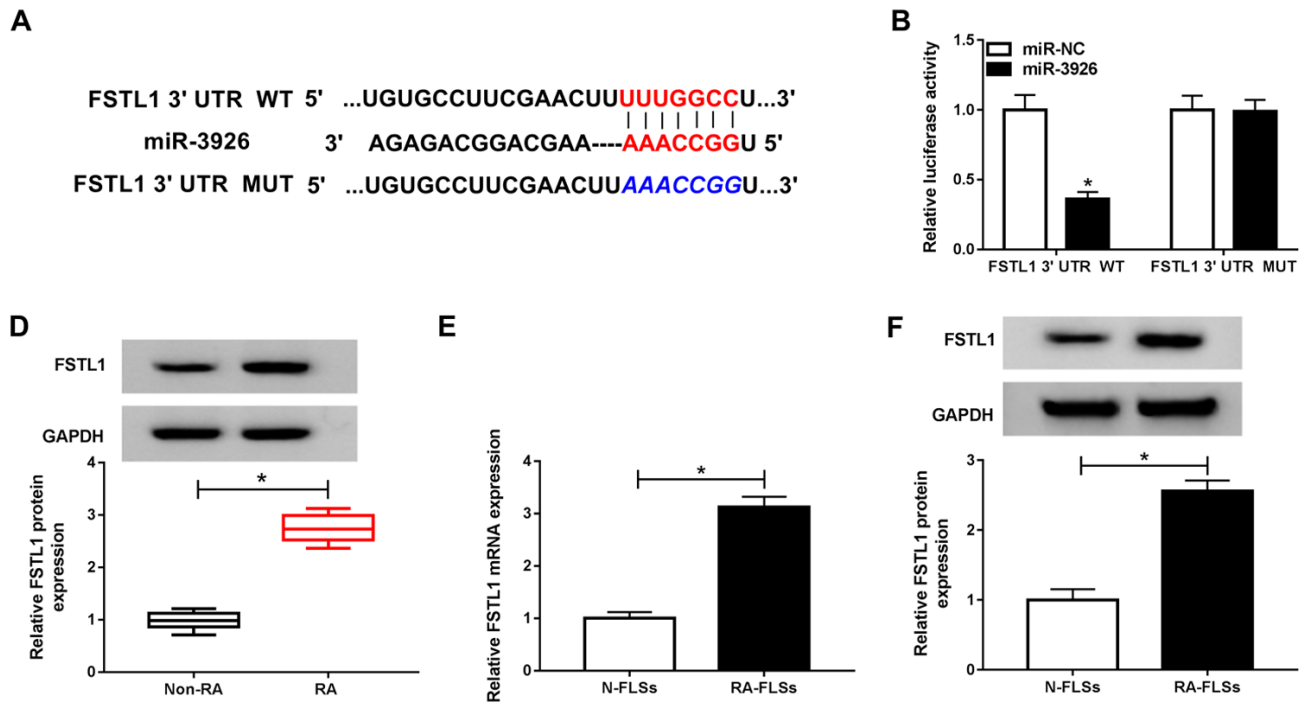
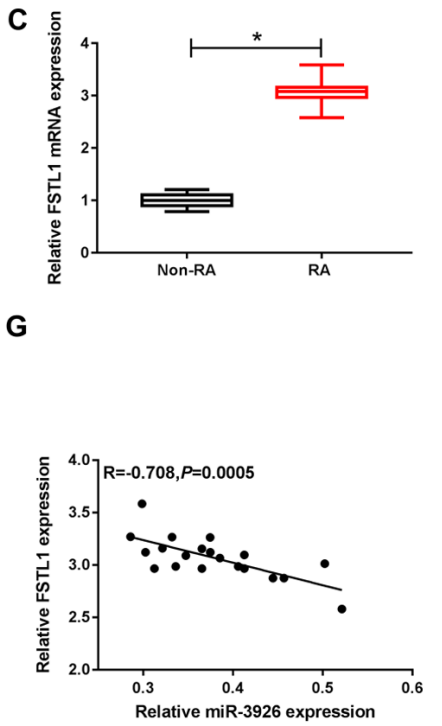

G
H

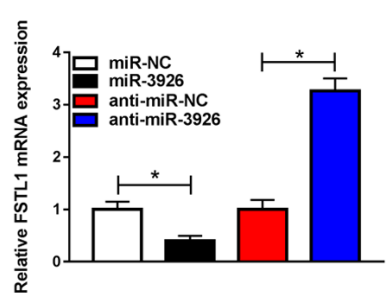

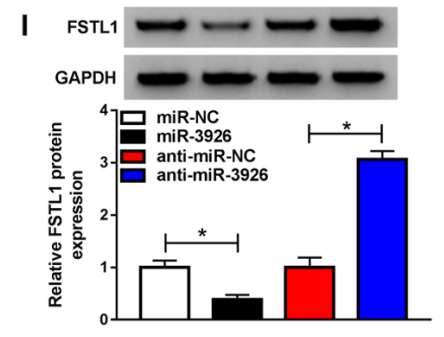

Figure 5. miR-3926 directly targets FSTL1 and negatively regulates its expression in RA-FLSs. (A) Potential binding sites between miR-3926 and FSTL1 were predicted via TargetScan. (B) Dual-luciferase reporter assay was performed to determine luciferase activity in RA-FLSs co-transfected with FSTL1 3'UTR WT or FSTL1 3'UTR MUT and miR-3926 or miR-NC. (C) mRNA and (D) protein expression levels of FSTL1 in RA synovial and non-arthritic control tissue were analyzed via RT-qPCR and western blotting, respectively. (E) mRNA and (F) protein expression levels of FSTL1 in RA-FLSs and N-FLSs were assessed via RT-qPCR and western blotting, respectively. (G) Pearson's correlation analysis was utilized to analyze the correlation between miR-3926 and FSTL1 in RA synovial tissue. RA-FLSs were transfected with miR-NC, miR-3926, anti-miR-NC or anti-miR-3926 and the (H) mRNA and (I) protein expression levels of FSTL1 were determined via RT-qPCR and western blotting assay, respectively. " $\mathrm{P}<0.05$ vs. miR-NC. miR, microRNA; FSTL1, follistatin-like protein 1; UTR, untranslated region; WT, wild type; MUT, mutant; NC, negative control; RT-qPCR, reverse transcription-quantitative PCR; RA, rheumatoid arthritis; FLSs, fibroblast-like synoviocytes; N, normal.

via RT-qPCR and western blotting. The data demonstrated that the mRNA and protein expression levels of FSTL1 were significantly increased in RA synovial tissues compared with non-arthritic control tissues (Fig. 5C and D). Similarly, the mRNA and protein expression levels of FSTL1 were higher in RA-FLSs than those in N-FLSs (Fig. 5E and F). Pearson's correlation analysis indicated that there was an inverse correlation between the expression levels of FSTL1 and miR-3926 in RA synovial tissue (Fig. 5G). Furthermore, miR-3926 overexpression significantly suppressed the mRNA and protein expression levels of FSTL1 in RA-FLSs, whereas miR-3926 inhibition markedly promoted the mRNA and protein expression levels of FSTL1 in RA-FLSs (Fig. 5H and I). Taken together, these results indicated that miR-3926 targeted FSTL1 and negatively modulated FSTL1 expression levels in RA-FLSs.

Depletion of miR-3926 rescues the effects of FSTL1 knockdown on cell proliferation, apoptosis, migration, invasion and inflammatory cytokine production in RA-FLSs. Based on the aforementioned results, it was hypothesized that miR-3926 may regulate the development of RA via targeting FSTL1. Thus, si-NC, si-FSTL1, si-FSTL1 + anti-miR-NC or
si-FSTL1 + anti-miR-3926 were transfected into RA-FLSs to investigate the association between miR-3926 and FSTL1 in the regulation of RA progression. si-FSTL1 transfection significantly suppressed the mRNA and protein expression levels of FSTL1 in RA-FLSs, whereas anti-miR-3926 transfection partly overturned these effects (Fig. 6A and B). The MTT assay demonstrated that FSTL1 knockdown significantly suppressed the proliferation of RA-FLSs, whereas miR-3926 inhibition reversed this suppressive effect (Fig. 6C). Flow cytometric analysis indicated that apoptosis of RA-FLSs was significantly promoted by FSTL1 knockdown, whereas this promotion was abolished by the inhibition of miR-3926 in RA-FLSs (Fig. 6D). Furthermore, the protein expression levels of C-caspase-3 were significantly elevated in RA-FLSs transfected with si-FSTL1, whereas anti-miR-3926 transfection significantly abrogated this elevation (Fig. 6E). As indicated by the results of the Transwell assay, cell migration and invasion of RA-FLSs transfected with si-FSTL1 were hampered, whereas these effects were partially weakened following transfection with anti-miR-3926 (Fig. 6F and G). Western blotting revealed that FSTL1 knockdown led to a decrease in the expression levels of IL- 6, IL- $1 \beta$ and TNF- $\alpha$ in RA-FLSs, whereas miR-3926 depletion attenuated this 


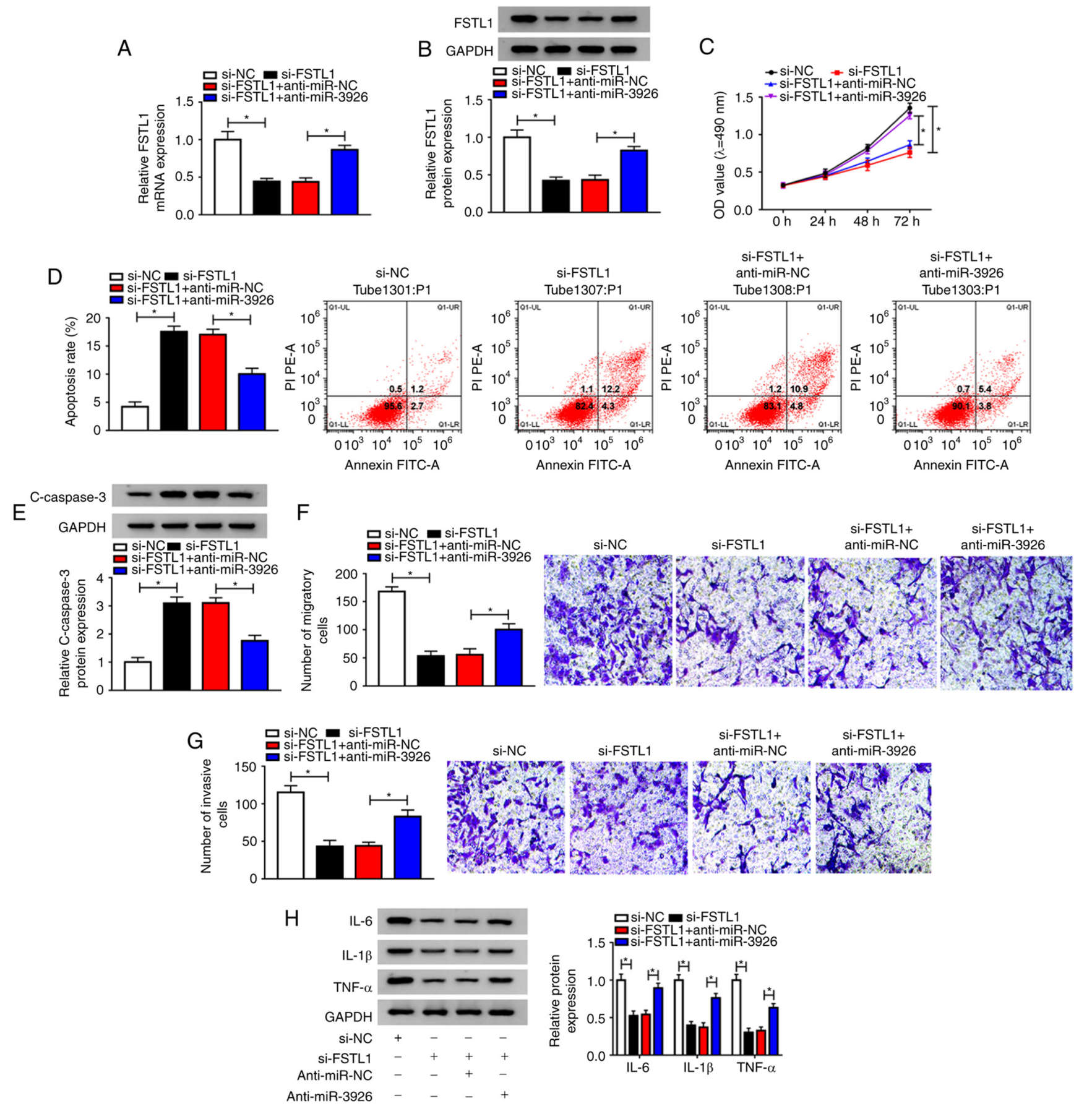

Figure 6. Inhibition of miR-3926 restores the inhibitory effect of FSTL1 knockdown on RA-FLSs development. RA-FLSs were transfected with si-NC, si-FSTL1, si-FSTL1 + anti-miR-NC or si-FSTL1 + anti-miR-3926. (A) mRNA and (B) protein expression levels of FSTL1 in RA-FLSs were determined via reverse transcription-quantitative PCR and western blotting, respectively. (C) Cell proliferation in RA-FLSs was assessed via MTT assay. (D) Cell apoptosis in RA-FLSs was evaluated via flow cytometric analysis. (E) Expression levels of C-caspase-3 in RA-FLSs were determined via western blotting. Cell (F) migration and $(\mathrm{G})$ invasion of RA-FLSs were determined by Transwell assay (magnification, $x 100$ ). (H) Expression levels of IL-6, IL-1 $\beta$ and TNF- $\alpha$ in RA-FLSs were assessed via western blotting. "P<0.05. miR, microRNA; FSTL1, follistatin-like protein 1; RA-FLSs, rheumatoid arthritis-fibroblast-like synoviocytes; si, small interfering RNA; NC, negative control; C-caspase-3, cleaved-caspase-3; IL, interleukin; TNF- $\alpha$, tumor necrosis factor- $\alpha$.

effect (Fig. 6H). These findings indicated that miR-3926 modulated cell proliferation, apoptosis, migration, invasion and inflammatory cytokine expression by interacting with FSTL1 in RA-FLSs.

ZFAS1 regulates FSTL1 expression levels via sponging miR-3926 in RA-FLSs. As indicated by Pearson's correlation analysis, FSTL1 expression levels were positively correlated with ZFAS1 expression levels in RA synovial tissue (Fig. 7A). In order to further determine the association between ZFAS1, miR-3926 and FSTL1 in RA-FLSs, si-NC, si-ZFAS1, si-ZFAS1 + anti-miR-NC or si-ZFAS1 + anti-miR-3926 was transfected into RA-FLSs, and RT-qPCR and western blotting were performed to measure the mRNA and protein expression levels of FSTL1, respectively. The data revealed that the mRNA and protein expression levels of FSTL1 were decreased in 
A

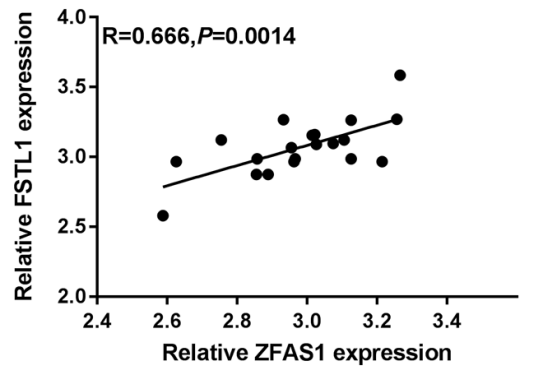

B

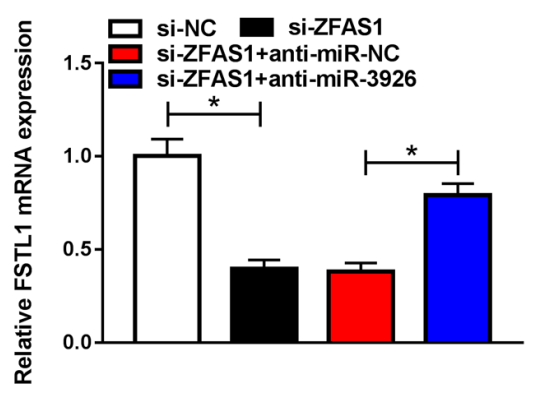

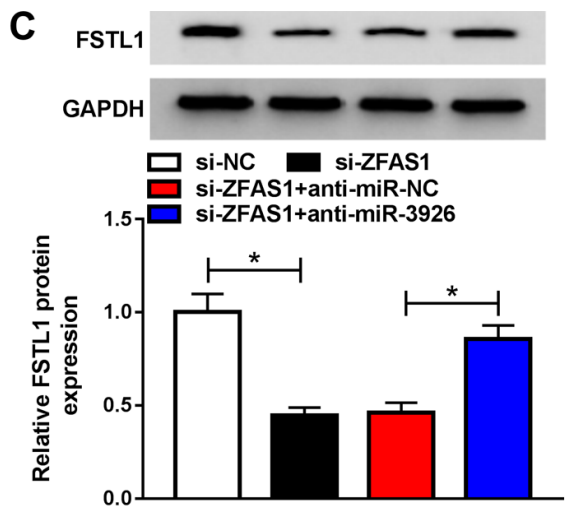

Figure 7. ZFAS1 positively modulates FSTL1 expression via miR-3926 in RA-FLSs. (A) Correlation between ZFAS1 and FSTL1 in RA synovial tissue was analyzed via Pearson's correlation analysis. (B) mRNA and (C) protein expression levels of FSTL1 in RA-FLSs transfected with si-NC, si-ZFAS1, si-ZFAS1 + anti-miR-NC or si-ZFAS1 + anti-miR-3926 were assessed via reverse transcription quantitative PCR and western blotting, respectively. ${ }^{\mathrm{P}}<0.05$. ZFAS1, zinc finger antisense 1; FSTL1, follistatin-like protein 1; miR, microRNA; RA-FLSs, rheumatoid arthritis-fibroblast-like synoviocytes; si, small interfering RNA; NC, negative control.

RA-FLSs transfected with si-ZFAS1, whereas anti-miR-3926 transfection partly rescued these effects (Fig. 7B and C). Taken together, these results indicated that ZFAS1 positively altered the expression levels of FSTL1 via sponging miR-3926 in RA-FLSs.

\section{Discussion}

Research is increasingly focusing on the association between IncRNAs and human disease development. The functional roles of ZFAS1 have been explored in numerous human diseases, such as non-small cell lung cancer (NSCLC) (22), T-cell acute lymphoblastic leukemia (23), hepatocellular carcinoma (24) and osteoarthritis (25). In the present study, ZFAS1 was significantly elevated in RA synovial tissues and RA-FLSs. Furthermore, ZFAS1 modulated the growth, apoptosis, migration, invasion and inflammation of FLSs via the miR-3926/FSTL1 axis in RA.

It has been confirmed that ZFAS1 mediates the regulation of cell growth, metastasis and apoptosis in a number of diseases. In NSCLC, ZFAS1 has been reported to be highly expressed, whereas silencing of ZFAS1 inhibited NSCLC cell growth via interacting with miR-193-3p (22). Furthermore, Ye et al (10) demonstrated that ZFAS1 was elevated in patients with RA, and contributed to RA-FLS migration and invasion by targeting miR-27a. In the present study, ZFAS1 was significantly elevated in RA synovial tissue and RA-FLSs. Depletion of ZFAS1 hampered RA-FLS growth, migration and invasion, and facilitated RA-FLS apoptosis. The present study also determined the impact of ZFAS1 knockdown on the expression of inflammatory cytokines. The data showed that the expression levels of IL- 6 , IL- $1 \beta$ and TNF- $\alpha$ were notably decreased following ZFAS1 knockdown in RA-FLSs, indicating that ZFAS1 knockdown inhibited inflammation in RA. Taken together, these data suggested that ZFAS1 may serve a key role in the progression of RA.

The interaction between IncRNAs and miRNAs has become a focus of research (25). In human diseases, ZFAS1 has been demonstrated to function as a sponge for multiple
miRNAs, such as miR-135a (26), miR-150 (27), miR-10a (28) and miR-329 (29). However, the association between ZFAS1 and miR-3926 has not been fully elucidated. To the best of our knowledge, the present study was the first to confirm that miR-3926 may be a direct target of ZFAS1. Wang et al (16) demonstrated that miR-3926 was decreased in RA and may be a biomarker for RA diagnosis. Fu et al (30) showed that miR-3926 was markedly decreased in RA, and that elevated expression levels of miR-3926 markedly suppressed RA synovial fibroblast growth and the secretion of inflammatory cytokines (IL-6, IL-1 $\beta$ and TNF- $\alpha$ ) by targeting Toll-like receptor 5. Consistent with these data, the present study demonstrated that miR-3926 expression levels were significantly lower and negatively regulated by ZFAS1 in patients with RA compared with healthy controls. Furthermore, transfection with miR-3926 led to notable decreases in RA-FLS growth, migration, invasion and inflammatory cytokine expression, as well as a marked enhancement in RA-FLS apoptosis, whereas these effects were all abrogated by pcDNA-ZFAS1 transfection.

Further analysis identified FSTL1 as a target gene of miR-3926. FSTL1 expression levels were increased in RA and inversely altered by miR-3926. Ehara et al (31) demonstrated that FSTL1 was overexpressed in RA. Mattiotti et al (32) revealed that high expression levels of FLST1 were associated with clinical features of RA. Clutter et al (33) showed that FSTL1 accelerated the progression of arthritis via increasing expression levels of IFN- $\gamma$. A study by Shi et al (19) discovered that FSTL1 was increased in RA; moreover, FSTL1 was targeted by miR-27a and was involved in the regulation of RA-FLS migration and invasion. The present study elucidated that deficiency of FSTL1 suppressed cell growth, migration, invasion and inflammation, and induced cell apoptosis in RA-FLSs, whereas miR-3926 inhibition attenuated these effects, indicating that miR-3926 may target FSTL1 to regulate the progression of RA.

In human diseases, ZFAS1 has been reported to act as a sponge for numerous miRNAs, such as miR-135a (26), miR-150 (27), miR-10a (28) and miR-329 (29). Further 
investigation is required to elucidate the associations between ZFAS1, FLST1 and these miRNAs in RA regulation.

In conclusion, the present study demonstrated that ZFAS1 was increased in patients with RA, and that ZFAS1 knockdown relieved the development of RA by modulating the miR-3926/FSTL1 axis. These results suggested that ZFAS1 may be a potential candidate for RA treatment.

\section{Acknowledgements}

Not applicable.

\section{Funding}

No funding was received.

\section{Availability of data and materials}

The datasets used and/or analyzed during the current study are available from the corresponding author on reasonable request.

\section{Authors' contributions}

PC and XY conceptualized the study and designed the experiments. JL and WZ collected and analyzed data. QW and MG performed the experiments. QW, PC and XY wrote, reviewed and edited the manuscript. QW and PC confirm the authenticity of all the raw data. All authors read and approved the final version of the manuscript.

\section{Ethics approval and consent to participate}

The present study was approved by the ethical review committee of the Second Hospital of Shandong University. Written informed consent was obtained from all enrolled patients.

\section{Patient consent for publication}

Not applicable.

\section{Competing interests}

The authors declare that they have no competing interests.

\section{References}

1. Araki Y and Mimura T: The mechanisms underlying chronic inflammation in rheumatoid arthritis from the perspective of the epigenetic landscape. J Immunol Res 2016: 6290682, 2016.

2. Torre LA, Bray F, Siegel RL, Ferlay J, Lortet-Tieulent J and Jemal A: Global cancer statistics, 2012. CA Cancer J Clin 65: 87-108, 2015

3. Bottini N and Firestein GS: Duality of fibroblast-like synoviocytes in RA: Passive responders and imprinted aggressors. Nat Rev Rheumatol 9: 24-33, 2013.

4. McInnes IB and Schett G: The pathogenesis of rheumatoid arthritis. N Engl J Med 365: 2205-2219, 2011.

5. Brennan FM and McInnes IB: Evidence that cytokines play a role in rheumatoid arthritis. J Clin Invest 118: 3537-3545, 2008.

6. Gibb EA, Brown CJ and Lam WL: The functional role of long non-coding RNA in human carcinomas. Mol Cancer 10: 38, 2011.

7. Mercer TR, Dinger ME and Mattick JS: Long non-coding RNAs: Insights into functions. Nat Rev Genet 10: 155-159, 2009.
8. Zou Y, Xu S, Xiao Y, Qiu Q, Shi M, Wang J, Liang L, Zhan Z, Yang X, Olsen N, et al: Long noncoding RNA LERFS negatively regulates rheumatoid synovial aggression and proliferation. J Clin Invest 128: 4510-4524, 2018.

9. Yue T, Fan X, Zhang Z, Liu Z, Guo M, Bai F, Gong X, Gao C and Xiao L: Downregulation of lncRNA ITSN1-2 correlates with decreased disease risk and activity of rheumatoid arthritis (RA), and reduces RA fibroblast-like synoviocytes proliferation and inflammation via inhibiting NOD2/RIP2 signaling pathway. Am J Transl Res 11: 4650-4666, 2019.

10. Ye Y, Gao X and Yang N: LncRNA ZFAS1 promotes cell migration and invasion of fibroblast-like synoviocytes by suppression of miR-27a in rheumatoid arthritis. Human Cell 31: 14-21, 2018.

11. Mendell JT and Olson EN: MicroRNAs in stress signaling and human disease. Cell 148: 1172-1187, 2012.

12. Furer V, Greenberg JD, Attur M, Abramson SB and Pillinger MH: The role of microRNA in rheumatoid arthritis and other autoimmune diseases. Clin Immunol 136: 1-15, 2010.

13. Philippe L, Alsaleh G, Pichot A, Ostermann E, Zuber G, Frisch B, Sibilia J, Pfeffer S, Bahram S, Wachsmann D and Georgel P: MiR-20a regulates ASK1 expression and TLR4-dependent cytokine release in rheumatoid fibroblast-like synoviocytes. Ann Rheum Dis 72: 1071-1079, 2013.

14. Wang L, Song G, Zheng Y, Wang D, Dong H, Pan J and Chang X: miR-573 is a negative regulator in the pathogenesis of rheumatoid arthritis. Cell Mol Immunol 13: 839-848, 2016.

15. Wang Y, Jiao T, Fu W, Zhao S, Yang L, Xu N and Zhang N: miR-410-3p regulates proliferation and apoptosis of fibroblast-like synoviocytes by targeting YY1 in rheumatoid arthritis. Biomed Pharmacother 119: 109426, 2019.

16. Wang W, Zhang Y, Zhu B, Duan T, Xu Q, Wang R, Lu L and Jiao Z: Plasma microRNA expression profiles in Chinese patients with rheumatoid arthritis. Oncotarget 6: 42557-42568, 2015.

17. Shibanuma M, Mashimo JI, Mita A, Kuroki T and Nose K: Cloning from a mouse osteoblastic cell line of a set of transfor ming-growth-factor-beta1-regulated genes, one of which seems to encode a follistatin-related polypeptide. Eur J Biochem 217: 13-19, 1993.

18. Chaly Y, Marinov AD, Oxburgh L, Bushnell DS and Hirsch R: FSTL1 promotes arthritis in mice by enhancing inflammatory cytokine/chemokine expression. Arthritis Rheum 64: 1082-1088, 2012.

19. Shi DL, Shi GR, Xie J, Du XZ and Yang H: MicroRNA-27a inhibits cell migration and invasion of fibroblast-like synoviocytes by targeting follistatin-like protein 1 in rheumatoid arthritis. Mol Cells 39: 611-618, 2016.

20. Aletaha D, Neogi T, Silman AJ, Funovits J, Felson DT, Bingham CO III, Birnbaum NS, Burmester GR, Bykerk VP, Cohen MD, et al: 2010 rheumatoid arthritis classification criteria: An American college of rheumatology/european league against rheumatism collaborative initiative. Arthritis Rheum 62: 2569-2581, 2010

21. Livak KJ and Schmittgen TD: Analysis of relative gene expression data using real-time quantitative PCR and the 2(-Delta Delta C(T)) method. Methods 25: 402-408, 2011.

22. Ge H, Chen S, Huang S and Zhu J: Long noncoding RNA ZFAS1 acts as an oncogene by targeting miR-193a-3p in human non-small cell lung cancer. Eur Rev Med Pharmacol Sci 23: 6516-6523, 2019.

23. Liu Q, Ma H, Sun X, Liu B, Xiao Y, Pan S, Zhou H, Dong W and Jia L: The regulatory ZFAS1/miR-150/ST6GAL1 crosstalk modulates sialylation of EGFR via PI3K/Akt pathway in T-cell acute lymphoblastic leukemia. J Exp Clin Cancer Res 38: 199, 2019.

24. Li T, Xie J, Shen C, Cheng D, Shi Y, Wu Z, Deng X, Chen H, Shen B, Peng C, et al: Amplification of long noncoding RNA ZFAS1 promotes metastasis in hepatocellular carcinoma. Cancer Res 75: 3181-3191, 2015.

25. Paraskevopoulou MD and Hatzigeorgiou AG: Analyzing miRNA-lncRNA interactions. Methods Mol Biol 1402: 271-286, 2016.

26. Zhao Z, Lin $\mathrm{X}$, Tong $\mathrm{Y}$ and Li W: Silencing lncRNA ZFAS1 or elevated microRNA-135a represses proliferation, migration, invasion and resistance to apoptosis of osteosarcoma cells. Cancer Cell Int 19: 326, 2019.

27. Wu T, Wu D, Wu Q, Zou B, Huang X, Cheng X, Wu Y, Hong K, Li P, Yang R, et al: Knockdown of long non-coding RNA-ZFAS1 protects cardiomyocytes against acute myocardial infarction via anti-apoptosis by regulating miR-150/CRP. J Cell Biochem 118: 3281-3289, 2017. 
28. Dong D, Mu Z, Wei N, Sun M, Wang W, Xin N, Shao Y and Zhao C: Long non-coding RNA ZFAS1 promotes proliferation and metastasis of clear cell renal cell carcinoma via targeting miR-10a/SKA1 pathway. Biomed Pharmacother 111: 917-925, 2019.

29. Wang JS, Liu QH, Cheng XH, Zhang WY and Jin YC: The long noncoding RNA ZFAS1 facilitates bladder cancer tumorigenesis by sponging miR-329. Biomed Pharmacother 103: 174-181, 2018.

30. Fu D, Xiao C, Xie Y, Gao J and Ye S: MiR-3926 inhibits synovial fibroblasts proliferation and inflammatory cytokines secretion through targeting toll like receptor 5. Gene 687: 200-206, 2019.
31. Ehara Y, Sakurai D, Tsuchiya N, Nakano K, Tanaka Y, Yamaguchi A and Tokunaga K: Follistatin-related protein gene (FRP) is expressed in the synovial tissues of rheumatoid arthritis, but its polymorphisms are not associated with genetic susceptibility. Clin Exp Rheumatol 22: 707-712, 2004.

32. Mattiotti A, Prakash S, Barnett P and van den Hoff MJB: Follistatin-like 1 in development and human diseases. Cell Mol Life Sci 75: 2339-2354, 2018.

33. Clutter SD, Wilson DC, Marinov AD and Hirsch R: Follistatin-like protein 1 promotes arthritis by up-regulating IFN-gamma. J Immunol 182: 234-239, 2009. 